\title{
Positive and negative symptoms in schizophrenia: A longitudinal analysis using latent variable structural equation modelling
}

Giuseppe Carràa $^{\mathrm{a}, \mathrm{b}}$, Cristina Crocamo ${ }^{\mathrm{b}}$, Matthias Angermeyer ${ }^{\mathrm{c}}$, Traolach Brugha ${ }^{\mathrm{d}}$, Mondher Toumi ${ }^{\mathrm{e}}$ and Paul Bebbington ${ }^{\mathrm{a}}$

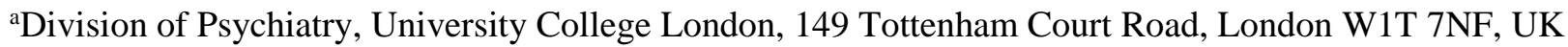
${ }^{b}$ Department of Medicine and Surgery, University of Milano Bicocca, Via Cadore 48, Monza 20900, Italy 'Department of Psychiatry, University of Leipzig, Johannisallee 20, 04137 Leipzig, Germany

${ }^{\mathrm{d}}$ Department of Health Sciences, University of Leicester, College of Medicine, Biological Sciences and Psychology, University of Leicester, Centre for Medicine, University Road, Leicester LE1 7RH,UK 'Laboratoire de Santé Publique, Université de la Méditerranée, Marseille, France

*Corresponding author at: Cristina Crocamo. Department of Medicine and Surgery, Via Cadore 48, Monza 20900, Italy. Tel. /Fax. +39 039 2332277. E-mail address: cristina.crocamo@unimib.it

Original article resubmitted for publication to Schizophrenia Research

Word count. Abstract: 236; Text body: 4716; Tables: 2; Figures: 1. Supplementary material: (Tables: 3; Figure: 1)

\begin{abstract}
Background: Recent network models of schizophrenia propose it is the consequence of mutual interaction between its symptoms. While cross-sectional associations between negative and positive symptoms are consistent with this idea, they may merely reflect their involvement in the diagnostic
\end{abstract} process. Longitudinal analyses however may allow the identification of possible causal relationships. The European Schizophrenia Cohort (EuroSC) provides data suitable for this purpose.

Methods EuroSC includes 1208 patients randomly sampled from outpatient services in France, Germany and the UK. Initial measures were repeated after 12 and 24 months. Latent variable structural equation modelling was used to investigate the direction of effect between positive and negative symptoms assessed with the Positive and Negative Syndrome Scale, controlling for the effects of depressed mood and antipsychotic medication.

Results The structural model provided acceptable overall fit $\left[\left(\chi^{2}(953)=2444.32, P<0.001 ; \mathrm{CFI}=0.909\right.\right.$; RMSEA $=0.046(90 \%$ CI: 0.043, 0.048); SRMR = 0.052]. Both positive and negative symptoms were persistent, and strongly auto-correlated. There were also persistent cross-sectional associations between positive and negative symptoms. While the path from latent positive to negative symptoms from T1 to T2 approached conventional levels of statistical significance $(P=0.051)$, that from T2 to T3 did not $(P=0.546)$. Pathways in the reverse direction were uniformly non-significant.

Conclusions There was no evidence that negative symptoms predict later positive symptoms. The prediction of negative symptoms by positive symptoms was ambiguous. We discuss implications for conceptualization of schizophrenic processes.

Keywords: Schizophrenia; Positive symptoms; Negative symptoms; Longitudinal studies; Diathesis models; Network models 


\section{INTRODUCTION}

The origins and course of schizophrenia are acknowledged to be complex. For over a century it has been conceived and diagnosed on the basis of distinctively characteristic symptoms, in particular delusions and hallucinations. In addition, since Kraepelin (tr. 1990) a conceptual and clinical contrast has been made between positive and negative symptoms. This distinction was strongly reasserted nearly 40 years ago (Strauss et al., 1974; Crow, 1980; Andreasen and Olsen, 1982), and negative symptoms have been included among the diagnostic criteria of schizophrenia since DSM-II (APA, 1968), remaining one of the five core dimensions in DSM-5 (APA, 2013).

The conceptual differentiation between the two symptom types has face validity, given that negative symptoms consistently load on at least two factors (diminished expression and anhedonia/asociality) that distinguish them from positive symptoms (Blanchard and Cohen, 2006). However, the relative diagnostic weight accorded to them has varied over time (Tandon et al., 2013), thereby affecting the propensity of the symptom types to be associated within the diagnostic category of schizophrenia (Galderisi et al., 2017). Notwithstanding, positive and negative symptoms are clearly associated, and the standard psychiatric view attributes this association to the expression of an underlying neuropathological diathesis (Walker, 1994; Seidman et al., 2016). In other words, negative symptoms may owe their origin to the same processes that produce positive symptoms (Pogue-Geile and Harrow, 1984). However, a diathesis account must deal with some inconvenient findings. First, schizophrenia characterised by salient negative symptoms (deficit schizophrenia) is more strongly correlated with neuro-anatomical abnormalities than non-deficit schizophrenia (Rowland et al., 2009). Secondly, both sorts of schizophrenia are thought to be associated with abnormalities of dopamine metabolism, but positive symptoms are more sensitive to medication targeting the dopamine system (Leucht et al., 2009, 2017). Third is the occurrence of positive and negative symptoms both across a range of clinical conditions and below the threshold of a diagnosable disorder (van Os and Reininghaus, 2016; Waters and Fernyhough, 2017; Bebbington and Freeman, 2017; Strauss and Cohen, 2017). Fourthly, when assessed in the early phases of illness, positive and negative symptoms show more than chance association, being roughly equally predictive of each other (Dominguez et al., 2010). Finally, the putative shared disease process must be variably expressed, given that symptoms emerge de novo and then fluctuate (Pogue-Geile and Harrow, 1984). Although this variability might be the consequence of an inherent biological rhythmicity, it seems equally likely to be due to variations in the psychosocial and pharmacological environment (Buchanan, 2007). Taken together, these findings imply that the causes of the two symptom types may not map perfectly onto each other. There are, however, alternatives to the diathesis account. Several authors have recently advocated a network model of psychopathology, whereby symptoms co-vary as a result of causal interactions between them, with no 
requirement for an underlying disease process (Borsboom and Cramer, 2013; McNally, 2016; Moffa et al., 2017). Symptoms may cause each other through a number of possible mechanisms including cumulative processes and feedback loops. For example, particular symptoms of schizophrenia may affect the social environment in ways that facilitate the expression of other symptoms. Thus, negative symptoms may encourage social withdrawal so that hallucinations and paranoid ideation become more pronounced (Wickham et al., 2014). Likewise, positive symptoms, for example extreme paranoia, may induce asociality and other facets of negative symptoms (Messinger et al., 2011). In addition, depressed mood may have a direct effect on both positive and negative symptoms (Fowler et al., 2012; Millan et al., 2014).

However, the possibility that negative symptoms are at least sometimes secondary has been conceded, since a basic distinction has been made between primary and secondary negative symptoms: it is the former that are thought to be the direct result of a putative disease process (Carpenter et al., 1988). Hence, secondary negative symptoms may be the consequence of unrelieved positive symptoms, the adverse effects of antipsychotics, or the social distortions brought about by the experience of having schizophrenia (Kirkpatrick et al., 2006). Nonetheless, apart from the sensitivity of social impoverishment to environmental improvement (Brown and Wing, 1970), changes in the level of these putative causes of secondary negative symptoms have little apparent influence on their subsequent course (Savill et al., 2015).

The possibility of mutual interactions is more meaningfully supported by evidence showing that primary negative symptoms are more common in people who have experienced positive symptoms at some point, resulting from the cumulative effect of positive symptoms. In addition, the two symptom types are less likely to be correlated when assessed cross-sectionally (Pogue-Geile and Zubin, 1987), than longitudinally (Tandon et al., 2013).

Investigation of the covariation of symptoms may therefore expand our understanding of the aetiology and course of schizophrenia. Unfortunately, such longitudinal studies have been rare, despite the long interval since the first reports on this topic, and their results have been inconsistent. One small study found that negative and positive symptoms were correlated at both the acute and six-month remission phases (Addington and Addington, 1991). Another reported that avolition and anhedonia predicted hallucinations, delusions and bizarre behaviour at 30-month follow-up (Kulhara and Chandiramani, 1990). In one 3-year follow-up investigation positive and negative symptoms appeared to increase together over time (Dollfus and Petit, 1995). Some of the inconsistency may relate to variable length of follow up: a more extended (10-year) follow-up study suggested that positive and negative symptom clusters are longitudinally independent (Eaton et al., 1995). Interestingly, a longitudinal study on the 
emergence of psychotic symptom types in general populations has shown that negative symptoms increased only after the emergence of positive symptoms (Werbeloff et al., 2015).

In addition, heterogeneity may play a key-role. In one study, changes in negative and positive symptoms were in general positively related for most of the combined latent trajectory classes identified; however, in some participants (who lacked readily identifiable features) positive subscale scores became increasingly predominant over negative scores (Chen et al., 2013).

It is clear that larger longitudinal studies are needed, consistent with the NIMH-MATRICS Consensus Statement on Negative Symptoms recommendation of more research on patterns of persistence of negative symptoms (Kirkpatrick and Fischer, 2006). The European Schizophrenia Cohort (EuroSC) was specifically set up to compare the attributes and correlates of diagnosed schizophrenia in large and representative cohorts from three European countries, France, Germany and the UK (Bebbington et al., 2005). Data were collected over a period of three years. It therefore provided a valuable opportunity to study the interplay between symptoms over time by applying latent variable (cross-lagged) structural equation modelling to three waves of assessment.

\subsection{ANALYTIC RATIONALE}

In this paper, we contrast four theoretical possibilities that might account for relationships between positive and negative symptoms of psychosis. The first is that negative symptoms are the consequence of positive symptoms, the second that the relationship operates in the reverse direction. The third proposes that the relationship is continuing and reciprocal i.e., that feedback loops exist between positive and negative symptoms. The final possibility is that the symptoms are associated because they have a common cause, the obvious candidate being an underlying pathophysiological diathesis. Longitudinal data may be capable of distinguishing between these four accounts. Consider data on negative and positive symptoms at three data-points. On their own, cross-sectional relationships between the symptom-types at each data point are uninformative about causal direction. However, cross-lagged relationships across either interval may indicate reciprocal effects or unique causal directions between the variables. Finally, auto-correlational relationships within symptom types assessed at successive data-points is consistent with persisting shared-cause models.

\section{EXPERIMENTAL/MATERIALS AND METHODS}

\subsection{Participants}

The EuroSC survey was a naturalistic two year follow-up of a cohort of people aged 18-64, with an established diagnosis of schizophrenia, and in contact with secondary psychiatric services, i.e., 
community outpatient services in line with national organizational standards, in nine community mental health catchment areas in France, Germany, and the UK. The principal objective was to identify and describe the types of treatment and patterns of care seen in people with schizophrenia, and to relate these to clinical outcomes, states of health, and quality of life. Local ethical approval for the study was obtained in each country. The settings, sampling strategies and inclusion/exclusion criteria are fully described elsewhere (Bebbington et al., 2005). The French samples were recruited from three centres in northern, central, and southern France, the German samples from two catchment areas in the former East Germany and two in the former West, and the British samples from Islington (in inner London) and the midland county of Leicestershire. Random sampling from lists of service users was adopted in all the French centres and in London, while an exhaustive inclusion strategy was used for the German sites and Leicestershire. Eligible patients were aged 18-64 years at the time of enrolment in the study, had a diagnosis of schizophrenia according to DSM-IV criteria, and had given signed informed consent. People were excluded if they had been hospitalized for the past 12 months, or were currently intoxicated, roofless or planning to leave the area (making follow-up assessment impracticable). In total, 1208 people with schizophrenia participated in the study, 288 in France, 302 in the UK, and 618 in Germany. Sixty-two percent of the sample were male, and the mean age was 40.8 years $(\mathrm{SD}=11.0$ years). The mean time since diagnosis was 15.7 years ( $\mathrm{SD}=9.8$ years).

\subsection{Measures}

An extensive battery of instruments was used to collect information during face-to-face interviews. Only those relevant to this study are presented here. In the UK and Germany, SCAN (Schedules for Clinical Assessment in Neuropsychiatry-version 1.0; WHO, 1992) was used to evaluate the 4-week period before interview and the most significant period of earlier psychopathology. Its component algorithm then allowed the establishment of research diagnoses of schizophrenia. In the French centres, schizophrenia was identified using the Structured Clinical Interview for DSM-IV (Spitzer et al., 1992). Information on symptom profile at the different time-points was based on the 30-item intervieweradministered, 7-point (1-7), Positive and Negative Syndrome Scale (PANSS) (Norman et al., 1996). Symptoms are rated in relation to the previous 72 hours. The PANSS was used in preference to alternatives because it was available in appropriate language versions. It also has the advantage of excluding secondary negative symptoms (Kay, 1990).

Depression levels were established using the Calgary Depression Scale for Schizophrenia (CDSS) (Addington et al., 1990, 1992). This consists of nine items, providing scores ranging from zero to 27. Information was also collected on antipsychotic medication.

\subsection{Procedures}


Individuals from the final list of participants were contacted consecutively by trained research assistants, who reassured them about privacy protection and sought their informed consent, with the help of local clinicians if needed. If they agreed, they were interviewed at home or in a clinical facility. The study was observational; no additional intervention was made either by, or at the behest of, the research team. The current analysis was based on assessments at baseline (Time 1: T1), and at 1- and 2year follow-up (T2 and T3). At T2, 962 participants completed the required measures, while 810 did so at T3. The initial and final assessments took around three hours, the intervening assessment somewhat less. Assessments sometimes required more than one session. All patients followed up were in treatment at T2 and T3. However, attrition analyses showed no significant differences in demographic factors or in any of the study variables between participants with data missing at any follow-up and the 810 participants who remained in the study. The main demographic and clinical characteristics at Time 1 are shown in table 1 . The mean scores for the psychotic symptoms and depression measures are shown in table 2. Overall, these scores declined with time, though not by much.

\section{TABLES 1 AND 2 ABOUT HERE}

\subsection{Analysis Plan}

Latent variable (cross-lagged) structural equation modelling (SEM) was used to test the plausibility of the hypothesized directional pathways leading from positive symptoms at time $t$, to negative symptoms at time $t+1$. We simultaneously examined the opposite directional pathways. Analyses were performed using Mplus 8 (Muthén and Muthén, 2017). Pre-analysis of the data did not reveal evidence of multivariate non-normality, and there was little missing data ( $2 \%$ of missing item responses across T1 to T3). We thus used the full information maximum likelihood estimation, which allowed us to handle missing outcome data by exploiting an embedded function of observed covariates and observed outcomes. Assuming missing at random and multivariate normality, this produces parameter estimates that are unbiased and more accurate and efficient than alternative imputation approaches, thereby maximizing statistical power (Little, 2013). Latent variable (cross-lagged) SEM involves estimation of a measurement model, and then a structural (cross-lagged) model, while accounting for measurement error (Little, 2013).

Thus, our initial model tested the measurement of the chosen (positive and negative) constructs by estimating factor loadings between at least two observed/measured indicators and underlying latent variables, as well as correlations between latent variables, using both exploratory and confirmatory factor analyses (Little, 2013).

First, we tested our PANSS data at T1 in order to explore how these were consistent with both standard and pentagonal models, since it has been claimed that the latter provides more accurate measurement 
model for clinical research (Kay et al., 1987, 2006; White et al., 1997). In particular, the standard model generates scales for Activation, Depression, Anergia, Thought Disturbance, and Paranoid/Belligerence, along with Positive and Negative. On the other hand, the pentagonal model, using 25 of the 30 PANSS items, is organized into five scales: Positive, Negative, Activation, Dysphoric Mood, and Autistic Preoccupation. Despite previous evidence about the significant overlap between the scales (i.e., Positive, Negative, Activation, Depression, Anergia, Thought Disturbance, Paranoid / Belligerence), in some cases those from the standard model (i.e., Anergia, Thought Disturbance, and Paranoid / Belligerence) did not match the pentagonal model closely. On the other hand, Autistic Preoccupation in the pentagonal model did not match the standard model. Nonetheless, although the pentagonal model seems to offer the best PANSS formulation, its authors point out that the factor structure and underlying model are complicated by the complexity of the disorder and its symptoms (Kay et al., 2006).

We thus ran preliminary Exploratory Factor Analyses (EFAs) for positive and negative constructs as measured in both models. We chose the Geomin rotation (Browne, 2001), which produces unbiased parameter estimates with good confidence interval coverage, as implemented in Mplus (Asparouhov and Muthén, 2009). For determining the number of factors and the items to be retained, we used scree plot analysis of eigenvalues above one (Costello and Osborn, 2005), together with four a priori criteria. These included: a) a primary factor loading greater than 0.40 , in order to maximize differentiation between factors (Ferguson and Cox, 1993); b) no or few item cross-loadings, as indicated by a difference of less than 0.2 between the highest and second highest factor loadings (Ferguson and Cox, 1993); c) each factor having at least three loadings above a pre-specified level to avoid the problem of common variance that arises when only two items are considered (Gorsuch, 1988); d) all retained items required to share the same conceptual meaning (Costello and Osborn, 2005). Since PANSS models have complex loading patterns, they are most suited to Confirmatory Factor Analyses (CFAs), and cannot be verified by exploratory methods of principal components or factor analysis (Kay et al., 2006). Once a suitable EFA solution was identified, we tested this factor structure with CFAs, using our sample data. Following conventional recommendations (Hu and Bentler, 1999), we report three goodness-of-fit-indices. As the chi-square statistic is dependent on sample size and likely to reject wellfitting models in moderately large samples such as ours (Byrne, 2011), we deemed it appropriate to use multiple measures of fit. The Comparative Fit Index (CFI) represents the extent to which the hypothesized model fits the data better than a null model. The Standardized Root Mean Square Residual (SRMR) signifies the standardized difference between observed and predicted correlations for the hypothesized model. Lastly, the Root Mean Square Error of Approximation (RMSEA) assesses the extent to which the hypothesized model fits the data. Values greater than .90 for the CFI, and less than 
0.08 for the SRMR and 0.05 for the RMSEA indicate that models fit the data acceptably (Hu and Bentler, 1999). CFAs were performed, assessing the temporal stability of the latent structure of the CFA model based on the maximum likelihood method of parameter estimation (Meade et al., 2005). If the model was rejected because of the associated estimated fit indices, it could be revised on an empirical basis with the Lagrange Multiplier (LM) test for adding further parameters to the model. The LM test indicates which parameters will significantly decrease $\chi^{2}$ and therefore enhance model fit. The set of parameters to be submitted for LM evaluation were additional factor loadings and correlated measurement errors. Successive modifications of the initial model were performed until the criterion for adequate model fit was achieved. Items were to be removed if LM results indicated noteworthy dual factor loadings.

In the second stage of analysis, we evaluated the structural (cross-lagged) model, retaining the components of the measurement model. This allows estimation of directional paths, both within (autoregressive: to establish their relative stability over time), and between (cross-lagged: to examine hypothesized and reverse directional pathways) latent variables. We thus tested whether latent positive symptoms would affect latent negative symptoms or vice versa. In addition, we used CDSS scores in the structural model as an observed time-varying covariate (predicting the latent variables assessed at the corresponding time point), in order to control for the effects of depression on the relationships between latent positive and negative symptoms. Similarly, adequate doses of antipsychotic medication, using dummy variables to distinguish monotherapy and combination therapy, were incorporated as a further observed time-varying covariate.

\section{RESULTS}

\subsection{Measurement Model}

Testing our PANSS data in order to explore consistency with both standard and pentagonal models, we ran preliminary Exploratory Factor Analyses for positive and negative constructs. However, these EFAs showed only modest correlations between positive and negative symptoms factors $(0.272$ and $0.266, \mathrm{p}<0.05$ for both), similar to those obtained in original factorial structure studies of PANSS standard and pentagonal models (Kay et al., 1987; White et al., 1997). In relation to PANSS items overall, the pentagonal solution was not supported, with different item structures making the interpretation of factors ambiguous. We thus focussed on the standard model for positive and negative scales. Examination of the scree plot of PANSS items relative to the positive and negative constructs found two factors with eigenvalues above one (Supplementary Figure 1). Confirmatory Factor Analyses actually suggested two dual loadings (in relation to P2: conceptual disorganization, and N7: stereotyped thinking), which we therefore removed sequentially from further analyses. Details on factor 
loadings are available in supplementary Table 1. The two extracted factors measured positive (P1, P3P7) and negative (N1-N6) symptoms. In order to control for possible systematic error arising from repeated assessments, the measurement error among the identical observed indicators of the latent variables (PANSS items) was allowed to be correlated over time (Newsom, 2015). We essentially assumed that errors of the same PANSS measure at different times are correlated, since this may account for the artefact of asking the same questions more than once. We tested this measurement model, which produced an acceptable fit $\left[\left(\chi^{2}(543)=2121.02, P<0.001 ; \mathrm{CFI}=0.922 ; \mathrm{RMSEA}=0.049\right.\right.$ (90\% CI: 0.047, 0.051); SRMR=0.060]. All factor loadings, ranging from 0.42 to 0.88 , were statistically significant $\left(P_{\mathrm{s}}<0.001\right)$, suggesting that all latent variables were adequately operationalized by the selected PANSS items.

\subsection{Structural Model}

We accordingly used this measurement model to test its structural equivalent. Holding depression levels and antipsychotic medication status as observed time-varying covariates (Fig. 1), the structural model provided acceptable overall fit $\left[\left(\chi^{2}(953)=2444.32, P<0.001 ; \mathrm{CFI}=0.909 ; \mathrm{RMSEA}=0.046(90 \%\right.\right.$ CI: 0.043, 0.048); SRMR=0.052]. Supplementary Table 2 shows standardized coefficients for positive to negative symptoms structural models, incorporating different combinations of time-varying covariates (Model 1, none; Model 2, CDSS scores only; Model 3, antipsychotic medication status only; Model 4, both). Depression emerged as an important factor, confirming the need for it to be taken into account.

Figure 1 shows the directional structural path between latent variables and relevant coefficients, as well as the within-time correlations. The structural path included, for both latent positive and negative symptoms, the specified first-order (from T1 to T2 and from T2 to T3) and second-order (from T1 to T3) autoregressive stability paths. Longitudinal paths for both positive and negative symptoms were all significant (T1 to T2; T2 to T3; and T1 to T3: .58, .56, .18, and .64, .49, .30, respectively). However, the relationships between adjacent measures were stronger than those between the first and the last measures.

The cross-sectional correlations between positive and negative symptoms were significant, albeit moderate $(\mathrm{T} 1=.25, \mathrm{~T} 2=.19$, and $\mathrm{T} 3=.28)$. Controlling for the time-varying covariates (depression and antipsychotic medication status), the cross-lagged paths linking latent positive and negative symptoms from T1 to T2 fell short of conventional statistical significance $(P=0.051)$, while those from T2 to T3 were very clearly non-significant $(P=0.546)$. More importantly, there was no support whatsoever for reverse directional pathways, i.e., from negative to positive symptoms, whether from $\mathrm{T} 1$ to $\mathrm{T} 2$ or from 
$\mathrm{T} 2$ to $\mathrm{T} 3$.

Finally, details for correlations among various latent variables and across time periods are shown in supplementary table 3.

\section{FIGURE 1 ABOUT HERE}

\section{DISCUSSION}

The EuroSC sample comprised people with established illness (average duration 16 years), in an established programmes of treatment, and with relatively low PANSS scores. Recently hospitalized subjects were excluded. Based on this large, representative cohort of people with a diagnosis of schizophrenia, followed up for a period of 2 years, our study has a number of strengths. Analyses of convenient non-interventional datasets are cost-effective, and have value in identifying possible links for further investigation.

Our retention of participants in the later time-points was good, but attrition inevitably tends to distort the representativeness of samples. The diagnosis of schizophrenia and the assessment of positive and negative symptoms was based on formal interviews. The longitudinal analysis represents a significant advance over cross-sectional studies, in which inferences about the directionality of effect must inevitably be extremely tentative. In order to provide a more robust test of links between positive and negative symptoms, we controlled for time-varying levels of depression and antipsychotic medication. Depressed mood might affect the level of positive symptoms directly (Fowler et al., 2012), and can mimic negative symptoms, while antipsychotic medication affects symptom levels differentially and hence the corresponding correlations (Sarkar et al., 2015). Dosages of antipsychotic medication were determined by the treating clinicians and analysed in broad categories: more detailed categorisation (Leucht et al., 2009) would have been difficult to interpret and of questionable benefit. For pragmatic reasons, the same raters at a given site generally carried out initial and follow-up interviews: they were therefore not blind to prior ratings although the intervals between interviews would have militated against accurate recall and hence bias. Evaluating the inter-site reliability of raters in three different languages was infeasible.

Overall, symptoms of all types declined in frequency over the two-year period. The modest size of this decline is consistent with the fact that participants were not chosen on the basis of a current acute episode. Findings relating to the links between positive and negative symptoms might have been more clear-cut in samples of people with first-episode schizophrenia, since medication would not have had 
its full impact on symptoms and course (Emsley et al., 2013), and sizeable fluctuations would be more likely. Furthermore, since we included for follow-ups only patients in treatment, symptom variation might have been attenuated, affecting cross-sectional and longitudinal correlations. Although we adopted full information maximum likelihood techniques, which generate plausible data based on observed outcomes and covariates, participants with minimal or worsened symptoms may not have been fully characterized, thus influencing results. Finally, we were unable to assess potential site effects either within or across countries: the limited number of clusters made multilevel analysis infeasible, since we would have more parameters than clusters.

The gold standard of causal inference is targeted intervention. Cross-lagged longitudinal models indicate merely whether a given factor is related to a different factor at a later date, and do not explicitly model the causes of change over time (Kenny, 2005). Nonetheless, we have to account for and interpret all the arrows in figure 1, whether significant or not. This includes the cross-sectional and cross-lagged relationships and the auto-correlations. How do they fit in with possible causal interpretations? Both types of symptom showed large and significant longitudinal self-correlation, although as the interval increased, the degree of fluctuation became greater, which is in agreement with the existing literature (Eaton et al., 1995; Chen et al., 2013; Kuipers et al., 2018). There were also appreciable cross-sectional associations between positive and negative symptoms at each time-point (Addington and Addington, 1991; Kulhara and Chandiramani, 1990; Dollfus and Petit, 1995). In the cross-lagged analyses, the lack of links between negative symptoms and subsequent positive symptoms offers no support either for a direct causal effect or for reciprocal feedback (Dominguez et al., 2010). However, the position regarding positive symptoms was ambiguous: they did not predict negative symptoms over the second interval, and the link over the first interval fell just short of conventional significance. It is clearly safest to assume that there was no longitudinal associations between positive symptoms and subsequent negative symptoms in either time period, despite the significant crosscorrelations between positive and negative symptoms at both T2 and T3. However, it might also reflect the attenuation of a genuine effect with the passage of time. Though the process is possibly selflimiting, positive symptoms may drive social amotivation (Jang et al., 2016). Such an effect is not implausible, as the two-year duration of the study is an appreciable period even in longstanding disorders. While longitudinal designs are persuasive, we cannot rule out confounding by unmeasured third variables, for instance comorbid dependence syndromes and medication compliance (Carrà et al., 2016). Moreover, interpretations in terms of an underlying diathesis for the association of positive and negative symptoms require an inference of such residual confounding from a persistent source. Our findings of very stable longitudinal auto-correlation and cross-correlation paths between symptoms, together with an absence of cross-lagged paths, are certainly consistent with a shared diathesis for 
positive and negative symptoms, whereas the tenuous link between positive symptoms at the first datapoint and negative symptoms at the second is not. We were unable to explore the area-level effects postulated to account for major differences in clinical characteristics of people with schizophrenia (Jongsma et al., 2018).

The salience of autocorrelation and cross-correlation in our results is also compatible with an alternative explanation. The concept of cause demands a temporal element, in that causes must be antecedent to effects. However, the appropriate period of effect is not intrinsically identifiable. The capacity to demonstrate cause in a longitudinal convenience sample is hostage to the assessment interval: if this is too long, effects may be masked, if too short, there may not have been time for the effect to occur. Experience sampling studies indicate that it is highly probable that causal interactions between psychological processes actually occur over periods of hours or days (Myin-Germeys et al. 2005; Delespaul et al., 2002). Such short-term effects may be cumulative, and remain apparent over longer periods (Fowler et al., 2012), but they may also be disguised in a combination of autocorrelation and cross-correlation.

Finally, causal inference would be furthered by research using other designs. Schizophrenia is defined primarily in terms of positive symptoms. Thus, the analysis of a cohort of individuals with established schizophrenia effectively guarantees the presence of positive symptoms but not of negative symptoms: this might privilege the effect of the former on the latter. There is therefore an argument for further studies of the mutual interaction of the two symptom types in longitudinal samples of the general population, or in high risk groups. In addition to clarifying the mechanisms underlying the illness (Kendler, 2016), a better understanding of the longitudinal interplay between positive and negative symptoms during the course of schizophrenia is important for designing effective treatment approaches. There may be potential value in treating existing positive symptoms and of preventing relapses as a way of improving (and limiting subsequent increases in) negative symptoms. A growing body of evidence suggests that positive symptoms unresponsive to vigorous pharmacological treatment may be improved through targeted cognitive-behavioural therapy (Kuipers et al., 2014; Garety et al., 2008), thereby promoting recovery and better long-term outcomes. 


\section{References}

- Addington, D., Addington, J., Maticka-Tyndale, E., Joyce, J., 1992. Reliability and validity of a depression rating scale for schizophrenics. Schizophr Res. 6 (3) 201-208.

- Addington, D., Addington, J., Schissel, B., 1990. A depression rating scale for schizophrenics. Schizophr Res. 3 (4) 247-251.

- Addington, J., Addington, D., 1991. Positive and negative symptoms of schizophrenia. Their course and relationship over time. Schizophr Res. 5 (1) 51-59.

- APA-American Psychiatric Association, 1968. DSM-II: Diagnostic and Statistical Manual of Mental Disorders. American Psychiatric Association, Washington DC.

- APA-American Psychiatric Association, 2013. Diagnostic and Statistical Manual of Mental Disorders (DSM-5®). American Psychiatric Association, Arlington.

- Andreasen, N.C., Olsen, S., 1982. Negative v positive schizophrenia. Definition and validation. Arch Gen Psychiatry 39 (7) 789-794.

- Asparouhov, T., Muthén, B., 2009. Exploratory Structural Equation Modeling. Struct Equ Modeling 16 (3) 397-438.

- Bebbington, P., Freeman, D., 2017. Transdiagnostic Extension of Delusions: Schizophrenia and Beyond. Schizophr Bull. 43 (2) 273-282.

- Bebbington, P.E., Angermeyer, M., Azorin, J.M., Brugha, T., Kilian, R., Johnson, S., Toumi, M., Kornfeld, A., EuroSc Research Group, 2005. The European Schizophrenia Cohort (EuroSC): a naturalistic prognostic and economic study. Soc Psychiatry Psychiatr Epidemiol 40 (9) 707-717.

- Blanchard, J.J., Cohen, A.S., 2006. The Structure of Negative Symptoms Within Schizophrenia: Implications for Assessment. Schizophr Bull. 32 (2) 238-245.

- Borsboom, D., Cramer, A.O.J., 2013. Network analysis: An integrative approach to the structure of psychopathology. Annu Rev Clin Psychol 9 91-121.

- Brown, G.W., Wing, J.K., 1970. Institutionalism and Schizophrenia: A Comparative Study of Three Mental Hospitals 1960-1968. Cambridge University Press, London.

- Browne, M.W., 2001. An Overview of Analytic Rotation in Exploratory Factor Analysis. Multivariate Behav Res. 36 (1) 111-150.

- Buchanan, R.W., 2007. Persistent negative symptoms in schizophrenia: an overview. Schizophr Bull. 33 (4) 1013-1022.

- Byrne, B.M., 2011. Structural equation modeling with Mplus: Basic Concepts, Application and Programming. Routledge, New York. 
- Carpenter, W.T., Heinrichs, D.W., Wagman, A.M., 1988. Deficit and nondeficit forms of schizophrenia: the concept. Am J Psychiatry 145 (5) 578-583.

- Carrà, G., Johnson, S., Crocamo, C., Angermeyer, M.C., Brugha, T., Azorin, J.M., Toumi, M., Bebbington, P.E., 2016. Psychosocial functioning, quality of life and clinical correlates of comorbid alcohol and drug dependence syndromes in people with schizophrenia across Europe. Psychiatry Res. 239 301-307.

- Chen, L., Johnston, J.A., Kinon, B.J., Stauffer, V., Succop, P., Marques, T.R., Ascher-Svanum, H., 2013. The longitudinal interplay between negative and positive symptom trajectories in patients under antipsychotic treatment: a post hoc analysis of data from a randomized, 1-year pragmatic trial. BMC Psychiatry 13320.

- Costello, A.B., Osborne, J.W., 2005. Best practices in exploratory factor analysis: Four recommendations for getting the most from your analysis. Practical Assessment Research \& Evaluation 10 (7) 1-9.

- Crow, T.J., 1980. Positive and negative schizophrenic symptoms and the role of dopamine. Br J Psychiatry 137 383-386.

- Delespaul, P., deVries, M., Van Os, J., 2002. Determinants of occurrence and recovery from hallucinations in daily life. Soc Psychiatry Psychiatr Epidemiol 37 (3) 97-104.

- Dollfus, S., Petit, M., 1995. Stability of positive and negative symptoms in schizophrenic patients: a 3-year follow-up study. Eur Psychiatry 10 (5) 228-236.

- Dominguez, M.D., Saka, M.C., Lieb, R., Wittchen, Hu., van Os, J., 2010. Early expression of negative/ disorganized symptoms predicting psychotic experiences and subsequent clinical psychosis: a 10-year study. Am J Psychiatry 167 (9) 1075-1082.

- $\quad$ Eaton, W.W., Thara, R., Federman, B., Melton, B., Liang, K.Y., 1995. Structure and course of positive and negative symptoms in schizophrenia. Arch Gen Psychiatry 52 (2) 127-134.

- Emsley, R., Oosthuizen, P., Koen, L., Niehaus, D., Martinez, L., 2013. Comparison of Treatment Response in Second Episode Versus First Episode Schizophrenia. J Clin Psychopharmacol 33 (1) $80-83$.

- Ferguson, E., Cox, T., 1993. Exploratory factor analysis: A users'guide. International Journal of Selection and Assessment 1 (2) 84-94.

- Fowler, D., Hodgekins, J., Garety, P., Freeman, D., Kuipers, E., Dunn, G., Smith, B., Bebbington, P.E., 2012. Negative cognition, depressed mood and paranoia: A longitudinal pathway analysis using structural equation modelling. Schizophr Bull 38 (5) 1063-1073.

- Galderisi, S., Färden, A., Kaiser, S., 2017. Dissecting negative symptoms of schizophrenia: History, assessment, pathophysiological mechanisms and treatment. Schizophr Res. 186 1-2. 
- Garety, P.A., Fowler, D.G., Freeman, D., Bebbington, P., Dunn, G., Kuipers, E., 2008. Cognitivebehavioural therapy and family intervention for relapse prevention and symptom reduction in psychosis: randomised controlled trial. Br J Psychiatry 192 (6) 412-423.

- Gorsuch, R.L., 1988. Exploratory Factor Analysis, In: Nesselroade, J.R., Cattell, R.B. (Eds.), Handbook of Multivariate Experimental, Plenum Press, New York, pp. 231-258.

- Hu, L., Bentler, P.M., 1999. Cutoff criteria for fit indexes in covariance structure analysis: Conventional criteria versus new alternatives. Struct Equ Modeling 6 (1) 1-55.

- Jang, S.K., Choi, H.I., Park, S., Jaekal, E., Lee, G.Y., Cho, Y., Choi, K.H., 2016. A Two-Factor Model Better Explains Heterogeneity in Negative Symptoms: Evidence from the Positive and Negative Syndrome Scale. Front Psychol 7707.

- Jongsma, H.E., Gayer-Anderson, C., Lasalvia, A., Quattrone, D., Mulè, A., Szöke, A., Selten, J.P., Turner, C., Arango, C., Tarricone, I., Berardi, D., Tortelli, A., Llorca, P.M., de Haan, L., Bobes, J., Bernardo, M., Sanjuán, J., Santos, J.L., Arrojo, M., Del-Ben, C.M., Menezes, P.R., Velthorst, E., Murray, R.M., Rutten, B.P., Jones, P.B., van Os, J., Morgan, C., Kirkbride, J.B.; European Network of National Schizophrenia Networks Studying Gene-Environment Interactions Work Package 2 (EU-GEI WP2) Group, 2018. Treated Incidence of Psychotic Disorders in the Multinational EUGEI Study. JAMA Psychiatry. 75(1) 36-46.

- Kay, S.R., 1990. Positive-negative symptom assessment in schizophrenia: psychometric issues and scale comparison. Psychiatr Q. 61 (3) 163-178.

- Kay, S.R., Fiszbein, A., Opler, L.A., 1987. The positive and negative syndrome scale (PANSS) for schizophrenia. Schizophr Bull 13 (2) 261-276.

- Kay, S.R., Hopler, L.A., Fiszbein, A., Ramirez, P.M., Opler, M., White, L., 2006. Positive and Negative Syndrome Scale (PANSS) Technical Manual. Multi-Health Systems, North Tonawanda.

- Kendler, K.S., 2016. Phenomenology of Schizophrenia and the Representativeness of Modern Diagnostic Criteria. JAMA Psychiatry 73 (10) 1082-1092.

- Kenny, D.A., 2005. Cross-lagged panel design. In: Everitt, B.S., Howell, D.C. (Eds), Encyclopedia of statistics in behavioral science, John Wiley \& Sons, Ltd, New York, vol. 1, pp. 450-451.

- Kirkpatrick, B., Fenton, W.S., Carpenter, W.T. Jr, Marder, S.R., 2006. The NIMH-MATRICS consensus statement on negative symptoms. Schizophr Bull 32 (2) 214-219.

- Kirkpatrick, B., Fischer, B., 2006. Subdomains Within the Negative Symptoms of Schizophrenia: Commentary. Schizophr Bull 32 (2) 246-249.

- Kraepelin, E., Quen, J.M., 1990. Psychiatry: A Textbook for Students and Physicians. Science History Publications, Canton. 
- Kuipers, J., Moffa, G., Kuipers, E., Freeman, D., Bebbington, P., 2018. Links between psychotic and neurotic symptoms in the general population: an analysis of longitudinal British National Survey data using Directed Acyclic Graphs. Psychol Med. doi: 10.1017/S0033291718000879. [Epub ahead of print]

- Kuipers, E., Yesufu-Udechuku, A., Taylor, C., Kendall, T., 2014. Management of psychosis and schizophrenia in adults: summary of updated NICE guidance. BMJ 348 g1173.

- Kulhara, P., Chandiramani, K., 1990. Positive and negative subtypes of schizophrenia. A follow-up study from India. Schizophr Res. 3 (2) 107-116.

- $\quad$ Leucht, S., Arbter, D., Engel, R.R., Kissling, W., Davis, J.M., 2009. How effective are secondgeneration antipsychotic drugs? A meta-analysis of placebo-controlled trials. Mol Psychiatry 14 (4) 429-447.

- Leucht, S., Leucht, C., Huhn, M., Chaimani, A., Mavridis, D., Helfer, B., Samara, M., Rabaioli, M., Bächer, S., Cipriani, A., Geddes, J.R., Salanti, G., Davis, J.M., 2017. Sixty Years of PlaceboControlled Antipsychotic Drug Trials in Acute Schizophrenia: Systematic Review, Bayesian MetaAnalysis, and Meta-Regression of Efficacy Predictors. Am J Psychiatry. doi: 10.1176/appi.ajp.2017.16121358.

- Little, T.D., 2013. Longitudinal structural equation modeling. The Guilford Press, New York.

- McNally, R.J., 2016. Can network analysis transform psychopathology? Behav Res Ther 8695 104.

- Meade, A.W., Lautenschlager, G.J., Hecht, J.E., 2005. Establishing measurement equivalence and invariance in longitudinal data with item response theory. International Journal of Testing 5 (3) 279-300.

- Messinger, J.W., Trémeau, F., Antonius, D., Mendelsohn, E., Prudent, V., Stanford, A.D., Malaspina, D., 2011. Avolition and expressive deficits capture negative symptom phenomenology: implications for DSM-5 and schizophrenia research. Clin Psychol Rev 31 (1) 161-168.

- Millan, M.J., Fone, K., Steckler, T., Horan, W.P., 2014. Negative symptoms of schizophrenia: clinical characteristics, pathophysiological substrates, experimental models and prospects for improved treatment. Eur Neuropsychopharmacol 24 (5) 645-692.

- Moffa, G., Catone, G., Kuipers, J., Kuipers, E., Freeman, D., Marwaha, S., Lennox, B.R., Broome, M.R., Bebbington, P., 2017. Using Directed Acyclic Graphs in Epidemiological Research in Psychosis: An Analysis of the Role of Bullying in Psychosis. Schizophr Bull. doi: 10.1093/schbul/sbx013.

- Muthén, L.K., Muthén, B.O., 2017 . Mplus User’s Guide. 1998-2017. Muthén and Muthén, Los Angeles, CA. 
- Myin-Germeys, I., Delespaul, P., Van Os, J., 2005. Behavioural sensitization to daily life stress in psychosis. Psychol Med 35 (5) 733-741.

- Newsom, J. T., 2015. Longitudinal structural equation modeling: A comprehensive introduction. New York: Taylor \& Francis. Chapter 5, pp. 122-151.

- Norman, R.M.G., Malla, A.K., Cortese, L., Diaz, F., 1996. A study of the interrelationship between and comparative interrater reliability of the SAPS, SANS and PANSS. Schizophr Res. 19 (1) 73-85.

- Pogue-Geile, M.F., Harrow, M., 1984. Negative and positive symptoms in schizophrenia and depression: a followup. Schizophr Bull 10 (3) 371-387.

- Pogue-Geile, M.F., Zubin, J., 1987. Negative symptomatology and schizophrenia: a conceptual and empirical review. Int J Ment Health 16 (4) 3-45.

- Rowland, L.M., Spieker, E.A., Francis, A., Barker, P.B., Carpenter, W.T., Buchanan, R.W., 2009. White matter alterations in deficit schizophrenia. Neuropsychopharmacology 34 (6) 1514-1522.

- Sarkar, S., Hillner, K., Velligan, D.I., 2015. Conceptualization and treatment of negative symptoms in schizophrenia. World J Psychiatry 5 (4) 352-361.

- Savill, M., Banks, C., Khanom, H., Priebe, S., 2015. Do negative symptoms of schizophrenia change over time? A meta-analysis of longitudinal data. Psychol Med 45 (8) 1613-1627.

- Seidman, L.J., Shapiro, D.I., Stone, W.S., Woodberry, K.A., Ronzio, A., Cornblatt, B.A., Addington, J., Bearden, C.E., Cadenhead, K.S., Cannon, T.D., Mathalon, D.H., McGlashan, T.H., Perkins, D.O., Tsuang, M.T., Walker, E.F., Woods, S.W., 2016. Association of Neurocognition With Transition to Psychosis: Baseline Functioning in the Second Phase of the North American Prodrome Longitudinal Study. JAMA Psychiatry 73 (12) 1239-1248.

- Spitzer, R.L., Williams, J.B., Gibbon, M., First, M.B., 1992. The Structured Clinical Interview for DSM-III-R (SCID). I: History, rationale, and description. Arch Gen Psychiatry 49 (8) 624-629.

- Strauss, G.P., Cohen, A.S., 2017. A Transdiagnostic Review of Negative Symptom Phenomenology and Etiology. Schizophr Bull 43 (4) 712-729.

- Strauss, J.S., Carpenter, W.T., Bartko, J.J., 1974. Part III, Speculations on the processes that underlie schizophrenic symptoms and signs. Schizophr Bull 1 (11) 61-69.

- Tandon, R., Gaebel, W., Barch, D.M., Bustillo, J., Gur, R.E., Heckers, S., Malaspina, D., Owen, M.J., Schultz, S., Tsuang, M., Van Os, J., Carpenter, W., 2013. Definition and description of schizophrenia in the DSM-5. Schizophr Res. 150 (1) 3-10.

- Walker, E.F., 1994. Developmentally moderated expressions of the neuropathology underlying schizophrenia. Schizophr Bull 20 (3) 453-480.

- Waters, F., Fernyhough, C., 2017. Hallucinations: A systematic review of points of similarity and difference across diagnostic classes. Schizophr Bull 43 (1) 32-43. 
- Werbeloff, N., Dohrenwend, B.P., Yoffe, R., van Os, J., Davidson, M., Weiser, M., 2015. The association between negative symptoms, psychotic experiences and later schizophrenia: a population-based longitudinal study. PLoS One 10 (3) e0119852.

- White, L., Harvey, P.D., Opler, L., Lindenmayer, J.P., 1997. Empirical assessment of the factorial structure of clinical symptoms in schizophrenia. A multisite, multimodel evaluation of the factorial structure of the Positive and Negative Syndrome Scale. The PANSS Study Group. Psychopathology 30 (5) 263-274.

- Wickham, S., Taylor, P., Shevlin, M., Bentall, R.P., 2014. The impact of social deprivation on paranoia, hallucinations, mania and depression: the role of discrimination social support, stress and trust. PloS One 9 (8) e105140.

- WHO-World Health Organization, 1992. SCAN:Schedule for Clinical Assessment in Neuropsychiatry. WHO Press, Geneva. 
Table 1. Sociodemographic and clinical characteristics at study entry of the study sample

\begin{tabular}{|l|c|}
\hline & $\mathbf{N}=\mathbf{1 2 0 8}$ \\
\hline Male & $743(62)$ \\
\hline Married/Living as couple & $254(21)$ \\
\hline Living condition & \\
Alone & $417(35)$ \\
With partner & $268(22)$ \\
With family & $295(24)$ \\
Collective accommodation & $141(12)$ \\
Homeless & $87(7)$ \\
\hline Ever worked & $1107(92)$ \\
\hline Age (years), mean (SD) & $40.76(10.97)$ \\
\hline Education (years), mean (SD) & $10.12(2.11)$ \\
\hline Age of illness onset (years), mean (SD) & $26.25(8.37)$ \\
\hline Length of illness (years), mean (SD) & $15.66(9.81)$ \\
\hline Overall illness course & \\
Single episode, full remission & $46(4)$ \\
Single episode, partial remission & $45(4)$ \\
Episode - no symptoms & $235(20)$ \\
Episode - residual symptoms & $500(42)$ \\
Continuous & $304(26)$ \\
Other or unspecified pattern & $61(5)$ \\
\hline With prominent negative symptoms & $395(33)$ \\
\hline Antipsychotic medication & \\
FGAs & $702(58)$ \\
SGAs & $291(24)$ \\
FGAs+SGAs & $159(13)$ \\
\hline Fis- FGAs) & \\
\hline
\end{tabular}

First- (FGAs) and second-generation (SGAs) antipsychotics

Values in parentheses are percentages except as otherwise indicated 
Table 2. Means and SDs for PANSS and CDSS total scores at different time periods

\begin{tabular}{|l|c|c|c|c|c|c|c|c|c|}
\hline & \multicolumn{3}{|c|}{ Time 1 (baseline) } & \multicolumn{3}{c|}{ Time 2 (12 months) } & \multicolumn{3}{c|}{ Time 3 (24 months) } \\
\hline & $\mathrm{N}$ & Mean & SD & N & Mean & SD & N & Mean & SD \\
\hline PANSS positive & 1188 & 12.39 & 5.57 & 949 & 11.84 & 5.24 & 800 & 11.31 & 5.26 \\
\hline PANSS negative & 1185 & 15.76 & 7.64 & 930 & 15.49 & 7.29 & 800 & 15.06 & 7.20 \\
\hline PANSS gen psych & 1179 & 29.33 & 10.68 & 942 & 28.16 & 9.53 & 797 & 26.57 & 9.15 \\
\hline CDSS & 1184 & 2.91 & 3.58 & 944 & 2.37 & 3.33 & 794 & 2.07 & 3.25 \\
\hline
\end{tabular}

PANSS, Positive and Negative Syndrome Scale; CDSS, Calgary Depression Scale for Schizophrenia

There are missing values for some items that SEM dealt with: the greatest numbers of missing items is for T3 CDSS 


\section{FIGURE LEGEND}

Fig. 1. Structural model of positive and negative symptoms with depression levels and antipsychotic medication status as observed time-varying covariates (standardized coefficients).

Ellipses represent latent variables. Rectangles represent observed (measured) time-varying covariates.

Single-headed and double-headed arrows represent the effect of one variable on another and within-time correlations between pairs of latent variables, respectively.

Dashed lines represent non-significant paths. T1 = baseline, T2 = 12 months follow-up; T3 = 24 months follow-up. ${ }^{*} \mathrm{P}<.05,{ }^{* *} \mathrm{P}<.01,{ }^{* * *} \mathrm{P}<.001$
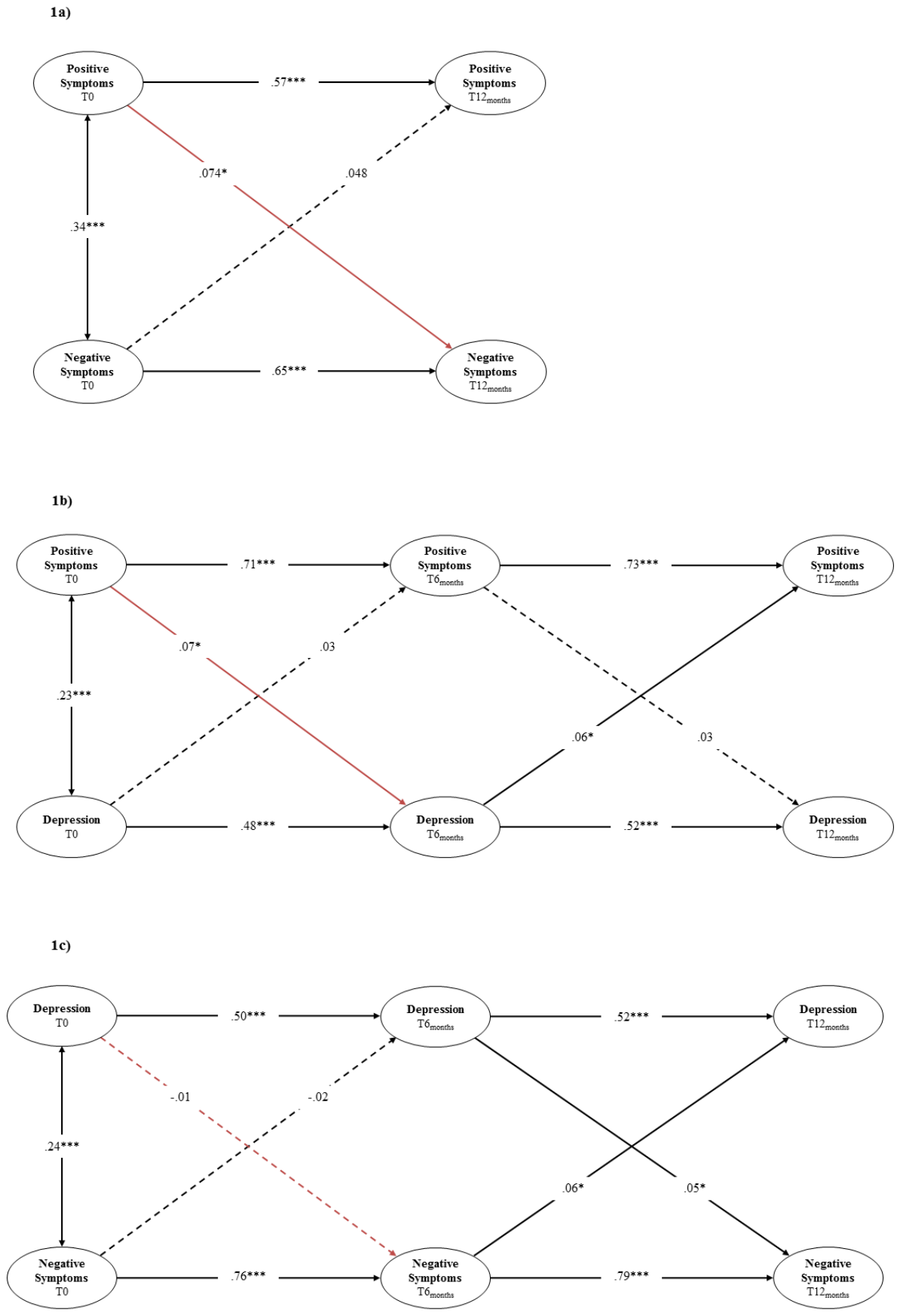
Fig. 2. Structural equation model for testing longitudinal indirect effects from positive to negative symptoms via depression levels with antipsychotic medication status as observed time-varying covariates (standardized coefficients). Ellipses represent latent variables. Rectangles represent observed (measured) time-varying covariates. Single-headed and double-headed arrows represent the effect of one variable on another and within-time correlations between pairs of latent variables, respectively. Dashed lines represent nonsignificant paths. T0 = baseline, T6 = 6 months follow-up; T $12=12$ months follow-up. $* \mathrm{P}<.05, * * \mathrm{P}<.01$, ***P $<0.001$

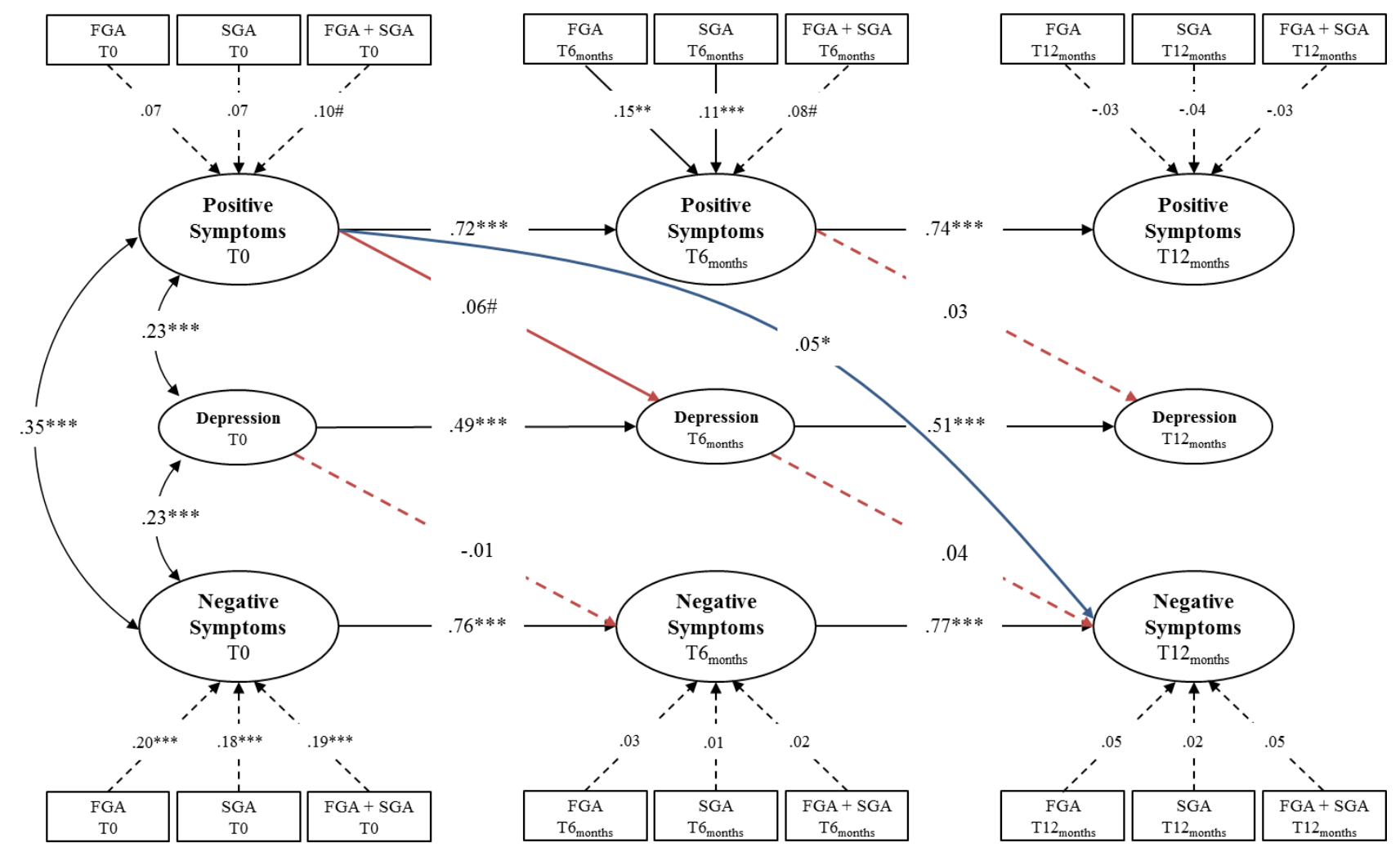




\section{Supplementary material}

\section{Supplementary (online-only) Figure 1.}

Scree plot PANSS items for positive and negative scales according to the standard model

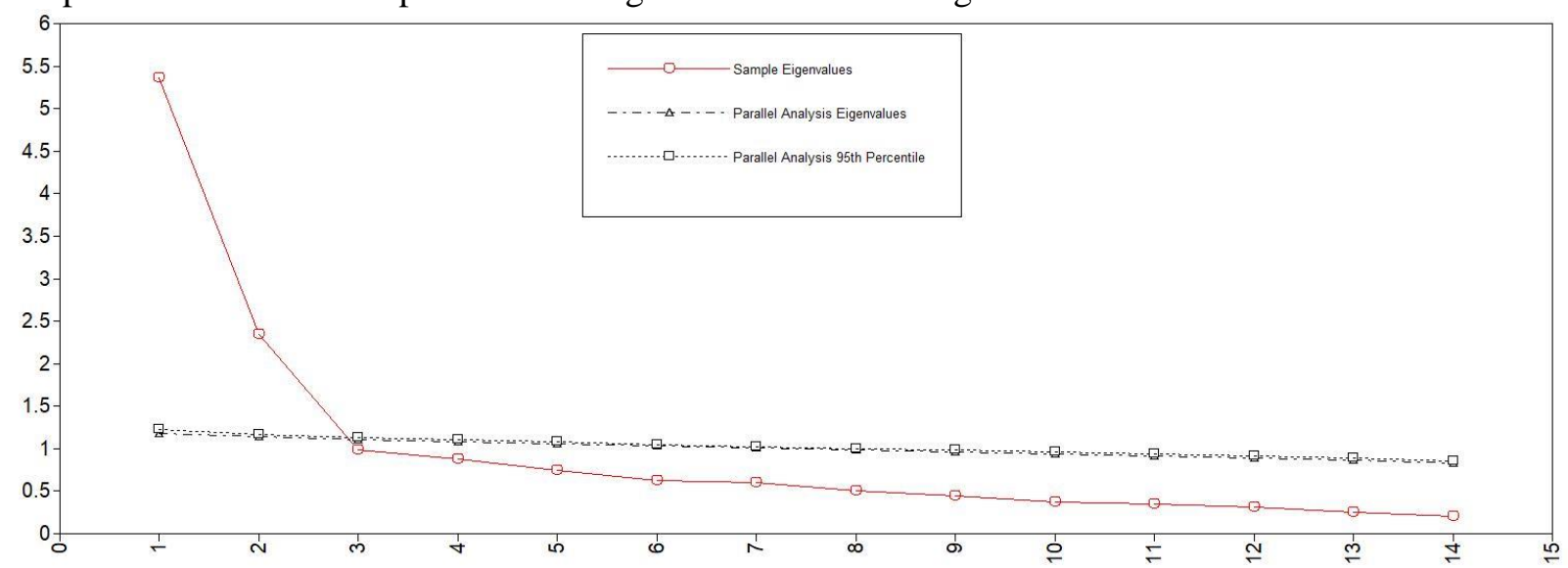

Supplementary (online-only) Table 1.

Factor loadings for the two extracted factors measuring PANSS positive and negative symptoms at time 1, preliminary analyses.

\begin{tabular}{|l|c|c|}
\hline Item & $\begin{array}{c}\text { Factor 1 } \\
\text { Positive }\end{array}$ & $\begin{array}{c}\text { Factor 2 } \\
\text { Negative }\end{array}$ \\
\hline p1 & 0.777 & -0.001 \\
\hline p2* & 0.563 & 0.331 \\
\hline p3 & 0.477 & -0.014 \\
\hline p4 & 0.547 & -0.015 \\
\hline p5 & 0.557 & -0.118 \\
\hline p6 & 0.569 & 0.161 \\
\hline p7 & 0.457 & 0.210 \\
\hline n1 & -0.065 & 0.863 \\
\hline n2 & -0.001 & 0.865 \\
\hline n3 & 0.033 & 0.852 \\
\hline n4 & 0.048 & 0.713 \\
\hline n5 & 0.215 & 0.437 \\
\hline n6 & -0.093 & 0.802 \\
\hline n7* & 0.417 & 0.435 \\
\hline
\end{tabular}

*dual loading 
Supplementary (online-only) Table 2.

Standardized coefficients for Positive to Negative symptoms structural models

\begin{tabular}{|c|c|c|c|c|}
\hline & Model 1 & Model 2 & Model 3 & Model 4 \\
\hline \multicolumn{5}{|l|}{$\begin{array}{l}\text { Correlations between } \\
\text { latent variables }\end{array}$} \\
\hline P1 with N1 & $.35^{* * *}$ & $.25^{* * *}$ & $.34^{* * *}$ & $.25^{* * *}$ \\
\hline P2 with N2 & $.27^{* * *}$ & $.19^{* * *}$ & $.27^{* * *}$ & $.19^{* * *}$ \\
\hline P3 with N3 & $.30^{* * * *}$ & $.28^{* * * *}$ & $.30^{* * * *}$ & $.28^{* * * *}$ \\
\hline \multicolumn{5}{|l|}{ Autoregressive paths } \\
\hline $\mathrm{P} 2$ on $\mathrm{P} 1$ & $.56^{* * *}$ & $.58^{* * *}$ & $.56^{* * *}$ & $.58^{* * *}$ \\
\hline $\mathrm{P} 3$ on $\mathrm{P} 2$ & $.55^{* * *}$ & $.56^{* * *}$ & $.56^{* * *}$ & $.56^{* * *}$ \\
\hline $\mathrm{P} 3$ on $\mathrm{P} 1$ & $.23^{* * *}$ & $.19^{* * *}$ & $.22^{* * *}$ & $.18^{* * *}$ \\
\hline $\mathrm{N} 2$ on $\mathrm{N} 1$ & $.65^{* * *}$ & $.64^{* * *}$ & $.65^{* * *}$ & $.64^{* * *}$ \\
\hline $\mathrm{N} 3$ on $\mathrm{N} 2$ & $.51^{* * *}$ & $.50^{* * *}$ & $.50^{* * *}$ & $.49^{* * *}$ \\
\hline $\mathrm{N} 3$ on $\mathrm{N} 1$ & $.31^{* * *}$ & $.30^{* * *}$ & $.32^{* * *}$ & $.30^{* * *}$ \\
\hline \multicolumn{5}{|l|}{ Cross-lagged paths } \\
\hline $\mathrm{N} 2$ on $\mathrm{P} 1$ & .06 & .07 & $.06^{*}$ & .07 \\
\hline $\mathrm{N} 3$ on $\mathrm{P} 2$ & .02 & .01 & .03 & .02 \\
\hline $\mathrm{P} 2$ on $\mathrm{N} 1$ & .04 & -.01 & .04 & -.01 \\
\hline $\mathrm{P} 3$ on $\mathrm{N} 2$ & .05 & .05 & .05 & .04 \\
\hline
\end{tabular}

Model 1: Positive to negative symptoms structural model;

Model 2: Positive to negative symptoms structural model with depression levels as observed time-varying covariate;

Model 3: Positive to negative symptoms structural model with antipsychotic medication status as observed time-varying covariate;

Model 4: Positive to negative symptoms structural model with depression levels and antipsychotic medication status as observed time-

varying covariates. ${ }^{*} \mathrm{P}<.05,{ }^{* *} \mathrm{P}<.01,{ }^{* * *} \mathrm{P}<.001$ 
Supplementary (online-only) Table 3. Correlation matrix for PANSS positive and negative constructs across time periods

\begin{tabular}{|l|c|c|c|c|c|c|}
\hline & P1 & P2 & P3 & N1 & N2 & N3 \\
\hline P1 & 1.00 & & & & & \\
\hline P2 & 0.60 & 1.00 & & & & \\
\hline P3 & 0.54 & 0.71 & 1.00 & & & \\
\hline N1 & 0.30 & 0.18 & 0.20 & 1.00 & & \\
\hline N2 & 0.27 & 0.29 & 0.27 & 0.67 & 1.00 & \\
\hline N3 & 0.24 & 0.23 & 0.36 & 0.64 & 0.71 & 1.00 \\
\hline
\end{tabular}

All correlations were significant at $\mathrm{p}<0.001$

PANSS: Positive and Negative Syndrome Scale

P1: PANSS positive at time 1; P2: PANSS positive at time 2; P3: PANSS positive at time 3;

N1: PANSS negative at time 1; N2: PANSS negative at time 2; N3: PANSS negative at time 3. 